\title{
Abordagem do Delirium na Comunidade
}

\section{An Overview of Delirium in the Community Setting}

\author{
Catarina Calheno Rebelo1*, Hugo Oliveira² ${ }^{1 *}$ Maria do Céu Rocha²
}

*Autor Correspondente/Corresponding Author Catarina Pereira Calheno Rebelo [catarina.pcr@hotmail.com] Rua de Alfredo Cunha 365, 4450-021 Matosinhos, Portugal

ORCID iD: 0000-0001-7563-0195

\section{RESUMO}

INTRODUÇÃO: O delirium é uma das complicações neuropsiquiátricas mais frequentes em Cuidados Paliativos. Constitui uma disfunção neurológica grave, não existindo diretrizes bem estabelecidas para a sua gestão na comunidade. O objetivo deste trabalho consiste na revisão da evidência existente sobre a abordagem do delirium na comunidade, em particular em Portugal.

MÉTODOS: Revisão da literatura utilizando os termos MeSH delirium, palliative care, domiciliary care, general practitioners, general physicians, primary care, Portugal e end of life.

RESULTADOS E CONCLUSÃO: O delirium é, habitualmente, multifatorial, tendo um impacto importante nos serviços de saúde, profissionais, cuidadores e, sobretudo, nos doentes. A evidência científica para o seu tratamento é escassa e aplica-se, sobretudo, aos cuidados hospitalares. A estruturação de cuidados de qualidade no âmbito da prevenção, identificação atempada e gestão precoce do delirium, no contexto dos Cuidados Paliativos na comunidade, deve incluir o doente, a sua família e o ambiente onde se insere.

PALAVRAS-CHAVE: Cuidados Paliativos; Delírio; Serviços de Assistência Domiciliar 


\section{ABSTRACT}

INTRODUCTION: Delirium is one of the most frequent neuropsychiatric complications in the Palliative Care setting. Despite being a severe neurological dysfunction, there are few studies describing its management in the community. Our objective was to review the existing evidence supporting best approaches to delirium in the community setting, considering the Portuguese context.

METHODS: Literature review using the following MeSH terms: "delirium", "palliative care", "domiciliary care", "general practitioners", "general physicians", "primary care", "Portugal" and "end of life".

RESULTS AND CONCLUSION: Delirium is usually multifactorial, impacting healthcare professionals, caregivers and, above all, patients. There is insufficient evidence concerning treatment of the condition, and available literature mostly concerns the hospital setting. In the Palliative Care setting, it is important that the patient, the family, and the environment are considered in order to assure early diagnosis and management of delirium by the family doctor.

KEYWORDS: Delirium; Home Care Services; Palliative Care

\section{INTRODUÇÃO}

O controlo de sintomas é uma das competências básicas dos Cuidados Paliativos (CP), que prestam apoio ao doente e à sua família, tendo como base a comunicação eficaz e o trabalho em equipa. ${ }^{1}$ Em Portugal, está prevista a implementação de CP comunitários, através de Equipas Comunitárias de Suporte em CP, integradas preferencialmente nos Agrupamentos de Centros de Saúde ou Unidades Locais de Saúde, assegurando a prestação de cuidados diretos aos doentes e famílias em situação de maior complexidade e vulnerabilidade. Estas equipas realizam procedimentos terapêuticos, como a administração de fármacos, e garantem o acompanhamento e tratamento de doentes que mantêm autonomia e capacidade para se deslocar aos locais de consulta, prestando igualmente apoio domiciliário a doentes em situação de dependência. ${ }^{2,3}$ Contudo, ainda existe uma escassez de serviços no âmbito dos CP em Portugal, uma vez que os existentes não alcançam todas as zonas do país. ${ }^{4}$

Os CP generalistas podem ser prestados pelos médicos de família (MF) na comunidade, caso detenham formação para tal, uma vez que mantêm um contacto próximo e frequente com doentes com doença incurável e progressiva. ${ }^{3}$ As equipas de CP na comunidade encontram-se numa posição central para prestar CP de qualidade, atempadamente, em articulação com muitos serviços prestadores de cuidados (tais como psicoterapia ou apoio social)..$^{5}$ De igual forma, os MF podem prestar CP a doentes no âmbito da consulta na unidade de saúde ou na consulta domiciliária. Um estudo realizado por Desmedt $M$ et al, que pretendia avaliar a coordenação de cuidados entre equipas de CP e os prestadores de Cuidados de Saúde Primários (CSP), concluiu que as equipas se mostravam muito satisfeitas com a disponibilidade, competência técnica e intervenção dos MF, na prestação de cuidados partilhados aos doentes integrados em CP. ${ }^{6}$

O delirium é uma das complicações neuropsiquiátricas mais frequentes em CP, quer em contexto hospitalar quer na comunidade. 'É uma disfunção neuropsiquiátrica grave, com alterações da consciência e cognição, de início súbito e carácter flutuante., ${ }^{7,8}$ Contudo, é uma síndrome subdiagnosticada e subtratada pelos profissionais de saúde, verificando-se uma escassez de estudos na área da gestão do delirium, particularmente no âmbito dos CP na comunidade. ${ }^{9,10}$ Em doentes com patologia crónica e avançada, o delirium apresenta um prognóstico desfavorável, estando associado a um aumento da morbimortalidade, ${ }^{11,12}$ maior declínio funcional e cognitivo, aumento do tempo de internamento, maior probabilidade de institucionalização e elevados custos associados. ${ }^{10,13} \mathrm{O}$ delirium apresenta um impacto importante ao ser gerador de sofrimento para o doente, família, cuidadores e profissionais de saúde, perturba a comunicação doente-família e aumenta a carga do cuidador. ${ }^{14,15}$ Adicionalmente, o custo associado a cuidados de saúde, um ano após alta, em doentes com delirium durante a hospitalização, é muito elevado. ${ }^{16}$ As intervenções realizadas para prevenir o delirium parecem ser custo-efetivas, apesar da evidência limitada. ${ }^{17}$

A gestão do delirium é desafiante nos diferentes serviços de prestação de cuidados e populações nas quais ocorre, mas o contexto único dos CP exige um reconhecimento e abordagem precoces, dada a vulnerabilidade 
da população, o seu status cognitivo e as implicações que o delirium pode ter na prestação de cuidados. $\bigcirc$ uso frequente de fármacos que podem conduzir a esta condição nos CP e o delirium no contexto de fim de vida são outros aspetos que tornam esta entidade particular no contexto dos CP. ${ }^{18}$ O delirium na comunidade implica uma gestão particular, quer pela capacidade de deteção, quer pelas opções terapêuticas disponíveis. Os estudos sugerem que a maioria das pessoas que sofre de doenças crónicas e avançadas prefere permanecer em casa até à sua morte, ${ }^{19,20}$ o que pode reforçar a pertinência da aquisição de competências nesta área. Este é, assim, mais um argumento para tornar premente a otimização de CP administrados por profissionais dos CSP.21,22

\section{MÉTODOS}

Foi realizada uma pesquisa bibliográfica nas bases de dados médicas PubMed e Cochrane Library, utilizando os termos MeSH delirium, palliative care, domiciliary care, general practitioners, general physicians, primary care, Portugal e end of life. A pesquisa incluiu artigos publicados em Inglês e Português. Os autores realizaram, também, uma pesquisa em cascata com consulta de livros, Normas de Orientação Clínica, repositórios de teses de mestrado e revistas portuguesas com o termo delirium, para identificar trabalhos na área, em Portugal. Desta pesquisa inicial resultaram 302 artigos, tendo sido selecionados para integrar a revisão, dada a sua pertinência, 61 artigos.

\section{RESULTADOS E DISCUSSÃO DEFINIÇÃO}

A definição de delirium tem-se transformado ao longo do tempo, refletindo a evolução na compreensão da sua fisiopatologia. É uma das complicações neuropsiquiátricas mais frequentes, cujo diagnóstico nem sempre é realizado, principalmente em doentes mais jovens. ${ }^{10}$

O diagnóstico do delirium é clínico e os seus critérios, de acordo com o Diagnostic and Statistical Manual of Mental Disorders (DSM-5) são ${ }^{23}$ :

- Perturbação da atenção (ex. capacidade reduzida de dirigir, focar, manter ou mudar a atenção) e do conhecimento (reduzida orientação para o ambiente);

- O distúrbio desenvolve-se num curto período de tempo (geralmente horas a alguns dias), representa uma alteração aguda da atenção e conhecimento de base e tende a flutuar em gravidade durante o dia;

- Distúrbio adicional na cognição (ex. défice de memó- ria, desorientação, linguagem, capacidade visuoespacial ou perceção);

- Os distúrbios dos critérios A e C não são melhor explicados por uma doença neurocognitiva pré-existente, estabelecida ou em evolução, e não no contexto de um nível gravemente reduzido de vigília, como no coma;

- Há evidência da história, exame físico ou dados laboratoriais, de que o distúrbio é uma consequência fisiológica direta de outra condição médica, intoxicação ou privação de uma substância (ex. devido a uma droga ou medicação), exposição a uma toxina ou é causada por etiologias múltiplas.

A definição de delirium da DSM-5, mais utilizada na prática clínica, baseia-se em perturbações da cognição como défices de memória, desorientação, linguagem, capacidade visual e perceção. Adicionalmente, perturbações da atenção (diminuição da capacidade de focar, manter e mudar a atenção) e vigília (reduzida orientação para o ambiente) são aspetos cruciais. $\bigcirc$ delirium desenvolve-se ao longo de um curto período (horas a dias) e flutua em gravidade durante o decurso de um dia. ${ }^{24}$

\section{APRESENTAÇÃO CLÍNICA}

O delirium pode ser classificado como hiperativo, hipoativo ou misto, consoante o nível de atividade psicomotora e de vigília verificados. ${ }^{13,25} \mathrm{O}$ delirium hiperativo é caracterizado por confusão, agitação e possível presença de alucinações e hiperalgesia (devido a síndrome de privação de opioides). Por outro lado, o delirium hipoativo inclui confusão, sonolência, prostração e letargia. ${ }^{13,21,25}$ No contexto dos CP, o delirium hipoativo é o mais frequente, apesar de os doentes com delirium hiperativo serem os mais frequentemente identificados e tratados. Doentes com delirium hipoativo podem ser, erradamente, diagnosticados como tendo depressão ou fadiga. ${ }^{13}$

As perturbações da perceção podem também ser evidentes, com presença de alucinações e ilusões. As alterações ao nível da cognição manifestam-se através de défices de memória, desorientação, perturbações da linguagem (com disartria, disnomia, disgrafia e afasia). Verificam-se, igualmente, perturbações afetivas e neurológicas, como movimentos involuntários, nomeadamente asterixis e mioclonias. ${ }^{26}$

\section{PREVALÊNCIA}

A grande dificuldade verificada na avaliação da prevalência e incidência do delirium na população com doença avançada deve-se à variedade de instrumentos de rastreio, escalas e diversidade de terminologia utilizada (agitação, confusão mental, alterações cognitivas, entre outras). ${ }^{27}$ 
Os diferentes estudos realizados, que pretendem estudar a prevalência de delirium em contexto de CP, apresentam um largo intervalo de valores de prevalência e incidência, que varia de acordo com o tipo de população de CP estudada. Embora existam dados disponíveis para o contexto hospitalar, a prevalência do delirium na comunidade - cuidados no domicílio e instituições - é escassa. ${ }^{28} \mathrm{Um}$ estudo estabeleceu a prevalência do delirium na comunidade entre 4\% a 12\%, ${ }^{29}$ enquanto outro demonstrou uma prevalência de 14\% na comunidade se considerados indivíduos > 85 anos e, em unidades de cuidados de longa duração, entre 1\% a 60\%. ${ }^{9}$

A prevalência do delirium num estudo retrospetivo realizado em contexto de CP hospitalares e de hospice, realidade não presente em Portugal, foi de 0\% a 8,4\%, aumentando para 35,7\%-39,2\% quando tanto o delirium documentado, como as descrições sugestivas de delirium, foram consideradas. Verificou-se, igualmente, que o uso do termo delirium é infrequente tanto a nível hospitalar como da comunidade, o que pode ser uma barreira para o seu diagnóstico e deteção. ${ }^{30}$

A prevalência do delirium em CP em Portugal é desconhecida, uma vez que não existem estudos na área, embora possa ser considerada semelhante à dos parceiros europeus. $^{21}$

\section{FISIOPATOLOGIA}

Os mecanismos fisiopatológicos do delirium são pouco conhecidos e a sua etiopatologia parece ser multifatorial.7 A diminuição da atividade colinérgica acompanhada de aumento dopaminérgico é uma teoria atualmente aceite, suportada pela perturbação da atenção, um dos sintomas cardinais do delirium. A administração de fármacos anticolinérgicos e a eficácia de fármacos que bloqueiam a dopamina, como o haloperidol, no tratamento sintomático do delirium, permitem sustentar esta teoria. ${ }^{7,13}$

\section{ETIOLOGIA}

O delirium é, habitualmente, multifatorial, estando associado a vários fatores de risco, que se dividem em fatores predisponentes e precipitantes (Tabela 1). 7,9,31 Não obstante, a etiologia do delirium é identificada em menos de metade dos doentes com doença terminal. ${ }^{32}$

A investigação etiológica deve basear-se numa avaliação criteriosa e individualizada, orientada pelos objetivos terapêuticos e prognóstico do doente. ${ }^{33}$ Os médicos enfrentam, muitas vezes, um desafio no que diz respeito a quão exaustivamente devem procurar fatores precipitantes do delirium, principalmente em doentes que estão a progredir para a fase terminal da sua doença. ${ }^{18}$
Devem ser avaliadas as causas potencialmente reversíveis de delirium (ex. desidratação, fármacos) e irreversíveis (sépsis, falência de órgãos major), estado mental basal e suas flutuações. $\bigcirc$ exame físico, de forma a excluir infeção, desidratação e sinais de falência orgânica (ex. renal, pulmonar e hepática), deve ser rotineiramente realizado. ${ }^{21}$ Os fármacos que estão habitualmente implicados como fatores precipitantes do delirium nos CP incluem as benzodiazepinas, corticosteroides, anticolinérgicos e opioides. Estima-se que cerca de $70 \%$ dos episódios de delirium induzidos por fármacos resolvem após realização de rotação de opioides e descontinuação de outros fármacos. ${ }^{18}$

O delirium partilha fatores de risco com outras síndromes geriátricas, nomeadamente demência, depressão, má nutrição, úlceras de pressão, incontinência urinária, dor crónica, quedas e abuso de idosos. ${ }^{9}$ Num estudo realizado na comunidade, em idosos admitidos nos cuidados hospitalares, determinou-se que as infeções, os fármacos e os distúrbios hidroeletrolíticos constituem os principais precipitantes de delirium na comunidade em idades avançadas. ${ }^{34}$

Intervenções dirigidas ao tratamento de precipitantes potencialmente preveníveis podem impedir o surgimen-

TABELA 1: Fatores predisponentes e precipitantes de delirium. 9,36,37

\begin{tabular}{|c|c|}
\hline Fatores predisponentes & Fatores precipitantes \\
\hline $\begin{array}{l}\text { - Idade avançada (>65 anos) } \\
\text { - } \text { Sexo masculino } \\
\text { - Demência } \\
\text { - } \text { Depressão } \\
\text { Estado funcional prévio } \\
\text { - Inatividade } \\
\text { - Isolamento social } \\
\text { - História de quedas } \\
\text { - Comorbilidades } \\
\text { - Dor crónica } \\
\text { - Doença terminal de ór- } \\
\text { gão (cardíaca, pulmonar, } \\
\text { hepática, cerebral...) } \\
\text { - Alcoolismo } \\
\text { Compromisso do sistema } \\
\text { sensorial (visual/auditivo) } \\
\text { - Alteração da capacidade da } \\
\text { ingestão oral } \\
\text { - Delirium prévio } \\
\text { - Disfunção de órgão devido } \\
\text { a neoplasia }\end{array}$ & 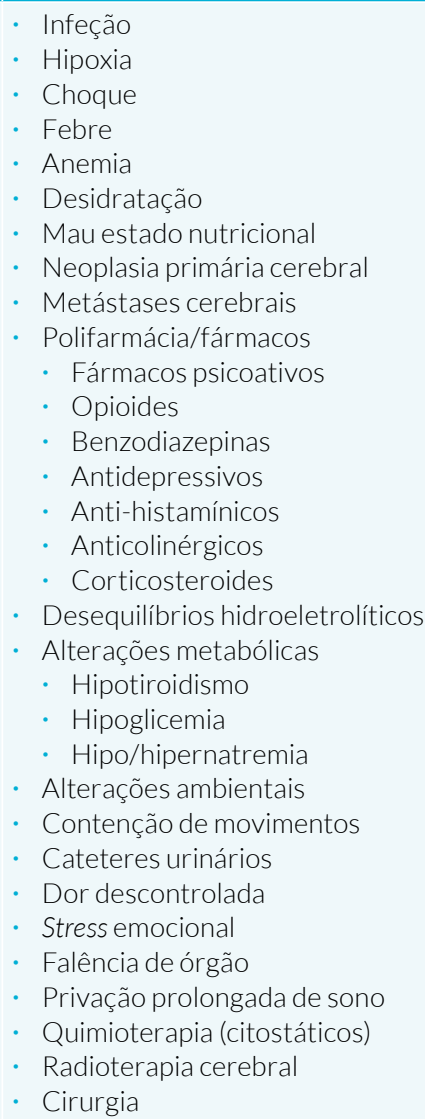 \\
\hline
\end{tabular}


to de delirium em idosos com demência. O conhecimento de fatores preditivos de delirium facilita o rastreio destes em idosos com risco aumentado, permitindo que os prestadores de cuidados de saúde mental previnam e identifiquem o início de um episódio. A evicção de fatores de risco de delirium conduz à diminuição direta da ocorrência deste e das suas consequências. ${ }^{35}$ Em cerca de 50\% dos casos, os quadros de delirium são reversíveis. ${ }^{25}$ Assim, os profissionais de saúde, os cuidadores e os familiares dos doentes devem ser treinados para reconhecer atempadamente e alertar para o surgimento de sintomas de delirium.?

O delirium persistente (perturbação cognitiva grave provocada pelo episódio de delirium), a idade mais jovem e também mais avançada, as hipoxemias e alterações metabólicas relativas a falência orgânica são fatores que contribuem para um pior prognóstico. $\bigcirc$ desenvolvimento de delirium em doentes com demência parece ser um preditor major de mortalidade em doentes em CP. ${ }^{11}$

\section{DIAGNÓSTICO}

O diagnóstico de delirium é clínico e deverá ser realizado o mais precoce possível. ${ }^{38}$ Um elevado nível de suspeição clínica é um fator chave para o diagnóstico desta entidade, sendo crucial a obtenção de informação por múltiplas fontes, nomeadamente o utente, os profissionais de saúde e a família. ${ }^{13}$ Os doentes em CP apresentam uma grande variabilidade individual, pelo que a avaliação do delirium deve ser personalizada e baseada na principal queixa do doente, história médica prévia e achados ao exame físico. ${ }^{31,39}$ É importante observar, pelo menos diariamente, os doentes admitidos em unidades de cuidados de longa duração e no domicílio, relativamente a alterações ou flutuações do comportamento habitual. Estas podem ser reportadas pelo doente, cuidador ou familiar e, nesse caso, um profissional treinado para reconhecer o diagnóstico de delirium deve avaliar clinicamente o doente de forma a confirmar o diagnóstico. $^{40-42}$

Orientações formais para o rastreio e abordagem do delirium têm sido extensivamente desenvolvidas em unidades de cuidados intensivos, podendo servir de modelo para outros contextos clínicos. ${ }^{43,44}$ Contudo, o reconhecimento do delirium permanece insuficiente na maioria dos contextos clínicos, incluindo naqueles em que CP ou de fim de vida serão, provavelmente, instituídos, como a comunidade. ${ }^{33}$ Existem vários desafios na investigação do delirium em contexto de cuidados na comunidade, em particular nos lares, nomeadamente: taxas de recrutamento baixas, vieses de exclusão, implicações em termos de recursos utilizados. ${ }^{45}$
O doente com suspeita de delirium deve ser avaliado, numa fase inicial, relativamente à sua função cognitiva. Se se verificar declínio cognitivo através da diminuição do score determinado através de métodos de rastreio de perturbação cognitiva, o profissional de saúde deve realizar o rastreio de delirium. ${ }^{7,21}$ Devem ser avaliados, igualmente, os fármacos administrados ao doente e possíveis interações e efeitos secundários dos mesmos a nível cognitivo, abuso de álcool e drogas, infeção, antecedentes de doença psiquiátrica, cirurgias ou internamentos recentes, alterações das circunstâncias ou ambiente. ${ }^{7}$

Os doentes com delirium hiperativo podem ser diagnosticados mais frequentemente tendo em conta os sintomas associados, que atraem mais atenção médica. ${ }^{38}$ Contudo, nem todos os doentes com agitação têm delirium - esta pode ser consequência de outros fenómenos (obstipação, retenção urinária, dor não controlada, ataques de pânico, mania). ${ }^{46}$

Um dos principais diagnósticos diferenciais de delirium é a demência. ${ }^{13}$ Pode ser difícil fazer esta distinção, principalmente porque muitos doentes apresentam ambas as patologias. Se existir incerteza quanto ao diagnóstico, o doente deve ser orientado inicialmente em relação ao quadro de delirium. ${ }^{40} \bigcirc$ delirium tem um início abrupto e um curso flutuante, enquanto a demência cursa, habitualmente, com um declínio lento e progressivo, cuja história pode ser obtida pela família. Ambas as condições apresentam declínio cognitivo, mas a demência pode ter um declínio mais acentuado e um dano mais grave do nível de consciência. Uma mudança de comportamento aguda é mais consistente com delirium, mesmo na presença de demência.,26 $\bigcirc$ delirium também apresenta outros diagnósticos diferenciais como a psicose e a depressão. ${ }^{7}$

Existem vários instrumentos para avaliar a função cognitiva do doente e para diagnosticar e monitorizar o delirium. A escolha do instrumento a utilizar deve ser ditada pelas psicométricas do instrumento, pelo estado geral do doente, pelo tempo disponível para o executar e pela experiência do médico. 8,47 Após identificado, este quadro clínico requer uma reavaliação contínua durante a permanência do doente no hospital, no domicílio ou no lar. 9

Não obstante, a aplicabilidade destes instrumentos em populações residentes em instituições de longa duração ou no domicílio é limitada, uma vez que não existem instrumentos validados para utilização na comunidade, pela ausência de estudos de investigação. ${ }^{14,21}$

O Mini-Mental State Examination (MMSE) é uma das ferramentas mais comummente utilizadas para avaliar 
declínio cognitivo, sendo utilizado no diagnóstico da demência, embora não se encontre recomendado para o diagnóstico de delirium. ${ }^{47,48} \bigcirc$ Confusion Assessment Method (CAM) é o instrumento mais efetivo e mais investigado no âmbito do delirium, sendo de utilização simples, validado para português, com uma duração de aproximadamente cinco minutos para completar e está validado para a população paliativa. ${ }^{13,21,47,49}$ O CAM também foi adaptado para outros contextos clínicos, como os lares. ${ }^{47,50}$ Existem outras escalas que podem ser utilizadas, embora não se encontrem validadas para utilização na comunidade. ${ }^{21}$

No contexto domiciliário, os cuidadores devem ser educados relativamente aos sinais e sintomas de delirium, assim como condições que possam indicar necessidade de avaliação imediata, por exemplo, alterações nos sinais vitais, dificuldade respiratória, dor torácica, hematúria e défices neurológicos focais recentes, entre outras. ${ }^{9}$ As equipas de enfermagem e os funcionários de lar ocupam, igualmente, uma posição privilegiada para detetar mudanças de comportamento devido ao contacto regular com os residentes, mas podem não estar treinados para reconhecer sintomas de delirium e tomar medidas apropriadas e atempadas. A deteção e gestão céleres do delirium podem prevenir contactos desnecessários com o MF ou admissões hospitalares evitáveis. ${ }^{17}$ Treinar os cuidadores/enfermeiras de lares e domicílios para usar o CAM e registar os resultados pode ser útil, assim como manter um registo do MMSE basal do doente, para fins comparativos. Uma melhor gestão e tratamento do delirium pode ser conseguido, assim, com melhoria do seu reconhecimento. ${ }^{30}$

No contexto dos CSP, a decisão principal consiste em determinar se a gestão de um doente em particular é realizada na comunidade ou se é imperativo referenciar o doente para os cuidados hospitalares. Esta decisão deve ser baseada numa avaliação holística do doente, tendo em conta a opinião dos seus familiares e/ou cuidadores. A falência da resposta ao tratamento inicial ou a necessidade de investigação adicional com meios complementares de diagnóstico não disponíveis nos CSP, podem justificar a referenciação para o hospital da área de residência do doente. Assim, uma avaliação laboratorial cuidadosa é, em muitos casos, uma parte importante da intervenção clínica, e inclui hemograma com leucograma, ionograma, função renal e hepática, análise sumária da urina, gasimetria (em doentes com dispneia, taquipneia, processo pulmonar agudo ou história de doença pulmonar significativa), radiografia do tórax, eletrocardiograma (em doentes com dor torácica, dispneia ou história de doença cardíaca) e análise cultu- ral apropriada. ${ }^{9,38,51}$ A tomografia axial computorizada cerebral pode ser útil em alguns casos, nomeadamente se o doente apresentar défices neurológicos focais de novo, história de traumatismo cranioencefálico ou febre associada a encefalopatia. Se há suspeita de eventos convulsivos, ou se a causa de delirium é desconhecida, a eletroencefalografia pode ser considerada. ${ }^{9}$

\section{TRATAMENTO}

A evidência científica para o tratamento do delirium é escassa e não está bem estabelecida, apesar de um número crescente de estudos emergirem nos últimos anos, com foco na sua abordagem e prevenção. O melhor tratamento desta condição é a prevenção, já presente e transversal à prática clínica do MF. O objetivo principal do tratamento de quadros de delirium consiste em reverter as suas causas, uma vez identificadas. ${ }^{38}$

A aplicabilidade dos estudos realizados noutros contextos clínicos ao contexto particular dos CP é, muitas vezes, limitada, pelo que uma interpretação criteriosa das recomendações deve ser tida em consideração. ${ }^{18}$ Diferentes estratégias de tratamento são necessárias para diferentes fatores precipitantes, tipos de delirium e patologia subjacente, variando, de igual forma, caso se trate de um doente em estadio avançado, nos últimos dias de vida ou numa fase precoce da sua doença. ${ }^{43}$ Desta forma, o nível de investigação e intervenção terapêutica num episódio de delirium é determinada pelo prognóstico estimado do doente e pelos objetivos estabelecidos de cuidados. ${ }^{36}$ Deve ser desenvolvido um plano de cuidados na comunidade individualizado, que aborde as patologias de forma independente, incluindo o delirium, recorrendo a tratamentos não farmacológicos e farmacológicos. ${ }^{9}$

A estruturação dos cuidados ao doente com delirium engloba não só o doente, mas também a sua família e o ambiente onde se insere. ${ }^{52}$ Antes da instituição de terapêutica farmacológica, o médico deve perceber as causas do quadro de delirium e o grau de distress provocado pelo mesmo. $\bigcirc$ tratamento farmacológico não deve ser a única abordagem instituída, devendo ser sempre completado por medidas não farmacológicas de suporte, mantendo o doente e os familiares informados do mesmo. ${ }^{8}$

\section{NÃO FARMACOLÓGICO}

As medidas não farmacológicas são importantes, principalmente para doentes vulneráveis, uma vez que minimizam a necessidade de administração de medicação (Tabela 2). ${ }^{10}$ Estas medidas podem ser instituídas na comunidade, orientadas pelo MF. A gestão de quadros clínicos como a desidratação e hipercalcemia, são aspetos igualmente importantes da intervenção. ${ }^{38}$ 
TABELA 2: Intervenções não farmacológicas para o tratamento do delirium. 7,36

\section{INTERVENÇÕES NÃO FARMACOLÓGICAS}

\section{DOENTE}

- Respeitar a dignidade do doente, mesmo perante atitudes inadequadas do mesmo

- Tratar o doente pelo seu nome

- Identificar sintomas prodrómicos de delirium (agitação, ansiedade, irritabilidade, desorientação, falta de atenção, perturbação do sono)

- Identificar as causas precipitantes

- Informar acerca do nome do médico e enfermeiro responsável aquando da visita

- Informar do momento do dia (manhã/tarde/noite)

- Estruturar as rotinas diárias com horas regulares e efetuar alterações mínimas no quarto

- Promover uma maior autonomia para as atividades de vida diárias:

- Incentivar a realização de tarefas simples (pentear-se, comer...) evitando ser demasiado exigente

- Estimular a memória retrógrada, de forma a restabelecer a confiança e orientação do doente

- Se o doente apresentar défices sensoriais deve ser assegurada a presença de óculos, aparelhos auditivos e próteses dentárias

- Promover a comunicação:

- Prestar atenção aos medos, angústias e possíveis alucinações do doente, de forma a superá-las

- Conversar, se possível, acerca das atividades diárias de forma clara e objetiva

- Atentar à linguagem não-verbal e à tonalidade afetiva da linguagem verbal, que pode ser percebida pelo doente

- Evitar conversas simultâneas

- Estimular a presença de membros da família/cuidadores, sempre que pertinente

- Promover o descanso noturno

AMBIENTE

- Seguro, confortável e tranquilo

- Colocar num local visível um calendário e um relógio

- Colocar ou manter objetos pessoais/familiares próximos do doente para preservar a orientação temporo-espacial

- Diminuir os estímulos

- Estabelecer contacto físico utilizando tom de voz suave

- Não permitir muitas pessoas no quarto e evitar vários doentes com delirium no mesmo quarto, no caso de lar/unidade de cuidados

- Promover a ventilação do quarto e garantir luz ténue

- Evitar as transferências de cama

- Evitar a utilização de grades na cama e outros meios de contenção físicos

FAMÍLIA

- Explicar sintomas e comportamentos do doente, assim como a conduta adequada a ter na presença do mesmo (aceitar a desorientação e o que o doente diz nesse contexto sem o tentar corrigir, contrariar ou desafiar)

- Promover a comunicação com o doente e com a equipa

- Esclarecer a alternância de períodos de lucidez e confusão e informar acerca do prognóstico

- Explicar as atitudes terapêuticas a instituir

- Explicar a eventual necessidade de internamento e o objetivo da sedação, caso seja necessária

- Realizar uma reunião familiar com a equipa, de forma a explicar os aspetos mencionados

As estratégias não farmacológicas de prevenção do delirium incluem medidas de orientação do doente e atividades terapêuticas, mobilização atempada e recorrente, minimização do uso de fármacos psicoativos, promoção de ciclos sono-vigília regulares, garantia de fácil acesso a equipamento de utilização pessoal (óculos, aparelhos auditivos) e prevenção da desidratação. ${ }^{9,21}$ De acordo com as recomendações da Direção Geral da Saúde, os doentes são elegíveis para a colocação de medidas de contenção quando manifestam comportamentos que o coloquem a si ou à sua envolvente em risco de sofrer danos, recusem tratamento compulsivo, nos termos legais, ou recusem tratamento vital, urgente. ${ }^{53}$

\section{FARMACOLÓGICO}

A terapêutica farmacológica do delirium está indicada para controlo sintomático, complementando as medidas não farmacológicas instituídas, assim como para prevenir que o doente se coloque em risco (Tabela 3). ${ }^{33}$ Se o doente adotar comportamentos disruptivos, como quedas, agitação intensa no leito ou remoção de dispositivos, deve ser rapidamente instituída terapêutica farmacológica, que constitui a primeira linha em doentes mais agitados. ${ }^{21}$ Por outro lado, não há evidência que os fármacos utilizados no tratamento do delirium consigam prevenir a sua ocorrência, pelo que não devem ser administrados profilaticamente. ${ }^{31}$

Os doentes sob CP devem ser tratados com doses baixas de fármacos, por períodos curtos, utilizando a via oral ou endovenosa. ${ }^{18}$ A via subcutânea é também muito utilizada no contexto de CP.7 Estes fármacos devem ser introduzidos após avaliação inicial e monitorização apropriadas. ${ }^{9}$ Fármacos que possam exacerbar quadros de delirium, como os anticolinérgicos, opioides e corticosteroides, devem ser suspensos após avaliação adequada. $^{7}$

O uso de antipsicóticos deve ser de último recurso no tratamento do delirium e não deve ser levado a cabo em doentes com delirium sem uma avaliação formal. ${ }^{9}$ Implica, assim, o envolvimento de profissionais especializados em CP.

O haloperidol é considerado tratamento de primeira linha para o delirium terminal por experts, embora estudos recentes não recomendem a utilização de neurolépticos no tratamento de delirium no contexto de CP.54,55 Num ensaio clínico randomizado realizado com doentes a receber CP, verificou-se que a avaliação individualizada dos precipitantes do delirium e estratégias de suporte instituídas resultam numa gestão mais eficaz do episódio de delirium e uma duração mais curta do mesmo, comparativamente à utilização de risperidona ou haloperidol. ${ }^{55}$

Os riscos associados à administração de fármacos antipsicóticos devem ser considerados. Os efeitos secundários extrapiramidais são os efeitos adversos mais co- 
TABELA 3: Intervenções farmacológicas para o tratamento do delirium na comunidade., ${ }^{7,21,56}$

\begin{tabular}{|c|c|c|}
\hline Fármaco & $\begin{array}{l}\text { Via de } \\
\text { administração }\end{array}$ & Dose \\
\hline Haloperidol & $\mathrm{PO} / \mathrm{SC} / \mathrm{IM} / \mathrm{IV}$ & $\begin{array}{l}\text { Inicial: 0,25 a } 1 \text { mg de } 8 / 8 \text { horas } \\
\text { ou 12/12 horas } \\
\text { Titulação: } 30 \text { em } 30 \text { minutos } \\
\text { Dose máxima } 20-30 \text { mg/dia }\end{array}$ \\
\hline Quetiapina & $\mathrm{PO}$ & $\begin{array}{l}\text { Iniciar com 12,5-25 mg 2x dia } \\
\text { - aumento de } 25-50 \mathrm{mg} / \text { dia até } \\
\text { dose de manutenção de 300- } \\
400 \mathrm{mg} / \text { dia } \\
\text { Dose máxima } 750-800 \mathrm{mg} / \mathrm{dia}\end{array}$ \\
\hline Olanzapina & $\mathrm{PO}$ & $\begin{array}{l}\text { 2,5-10 mg/dia, uma toma ao } \\
\text { deitar }\end{array}$ \\
\hline Risperidona & $\mathrm{PO}$ & $\begin{array}{l}\text { 0,25-0,5 mg/dia; 1-2 tomas/dia } \\
\text { Dose máxima } 6 \text { mg/dia }\end{array}$ \\
\hline Clorpromazina & $\mathrm{PO}$ & 12,5-150 mg/dia; $1-3$ tomas/dia \\
\hline $\begin{array}{l}\text { Levomeproma- } \\
\text { zina }\end{array}$ & $\mathrm{PO} / \mathrm{SC}$ & $\begin{array}{l}6,25 \text { até } 100 \mathrm{mg} / \mathrm{dia} ; 1-2 \\
\text { tomas/dia ou } \\
\text { perfusão SC contínua }\end{array}$ \\
\hline Midazolam & $\mathrm{PO} / \mathrm{IV} / \mathrm{SC}$ & $\begin{array}{l}\text { Oral: 0,5-2 mg 1/1 horas ou 4/4 } \\
\text { horas } \\
\text { SC: } 3-5 \text { mg 6/6h ou } 1 \text { mg/h em } \\
\text { perfusão } \\
\text { Dose máxima: } 30-80 \text { mg/24 } \\
\text { horas }\end{array}$ \\
\hline
\end{tabular}

IM - intramuscular; IV - intravenoso; PO - per os; SC - subcutânea.

Nota: As doses descritas dos fármacos apresentados são doses médias.

muns e verificam-se com os agentes típicos e atípicos. ${ }^{18}$ Outros efeitos adversos como sedação, efeitos anticolinérgicos, arritmias cardíacas e interações medicamentosas também devem ser considerados. ${ }^{8}$ Caso ocorram efeitos secundários como efeitos extra-piramidais, a olanzapina, a risperidona e a quetiapina podem substituir o haloperidol.7,56

Casos mais graves de delirium terminal, com agitação e agressividade associadas, podem ser geridos com midazolam ou levomepromazina. Esta abordagem terapêutica, juntamente com a administração de altas doses de opioides, requer experiência e pode gerar problemas éticos. Desta forma, a gestão destes doentes em conjunto com equipas de CP é essencial quando se estabelece um quadro de delirium. ${ }^{46} \mathrm{O}$ haloperidol, midazolam e levomepromazina encontram-se disponíveis em Portugal para administrar, em formulação injetável e oral, assim como a olanzapina oral. ${ }^{57}$

As benzodiazepinas e outros hipnóticos não devem ser utilizadas rotineiramente, limitando o seu uso a situações críticas em que os neurolépticos não podem ser usados e casos de delirium devido a abstinência alcoólica. Estas substâncias podem agravar, precipitar ou masca- rar o delirium, e resultar em efeitos adversos com stress substancial e sobrevida desfavorável para os doentes. Assim, eles devem apenas ser usados em doentes sintomáticos e a duração da terapêutica deve ser limitada., ${ }^{9,24}$ Paradoxalmente, num estudo preliminar com doentes hospitalizados com delirium associado a agitação no contexto de cancro avançado, a adição de lorazepam ao haloperidol, comparativamente com o haloperidol isoladamente, resultou numa redução significativamente superior da agitação ao final de 8 horas. ${ }^{58}$

Alguns estudos mostram benefício na utilização de agonistas alfa- 2 dexmedetomidina. ${ }^{44}$ Nenhum estudo avaliou a aplicabilidade destes fármacos no contexto da comunidade. Outros estudos salientam o risco associado ao uso de inibidores da colinesterase no tratamento do delirium, pelo que a utilização destes fármacos na comunidade não está estabelecida. ${ }^{18,59}$

\section{DELIRIUM HIPOATIVO}

No caso particular do delirium hipoativo deve ser tomada uma atitude expectante, mantendo atenção à flutuação com início rápido de agitação. A evidência científica para o seu tratamento sintomático é limitada, podendo mesmo agravar o estado geral do doente. 12,21,54

\section{CONCLUSÃO}

A confiança e a disponibilidade do MF são essenciais na articulação da prestação de CP, assim como no controlo sintomático e gestão de patologia não oncológica. ${ }^{60}$ Os profissionais dos CSP, tanto médicos como enfermeiros de família, encontram-se numa posição privilegiada para iniciar CP Generalistas precoces. Contudo, verificaram-se vários desafios no que diz respeito à atuação do MF, nomeadamente ao nível da gestão das expetativas do doente e da sua família, conflitos familiares e falta de planeamento e conhecimentos acerca de cuidados avançados. ${ }^{60}$

Um estudo identificou algumas barreiras na prestação de cuidados ao doente com delirium: falta de conhecimentos e experiência na área dos $\mathrm{CP}$, desconhecimento e imprevisibilidade do curso da doença, falha na identificação de utentes que necessitem de CP, atraso na perceção de necessidade de cuidados paliativos em doentes não oncológicos, falhas de comunicação com os doentes, comunicação e/ou cooperação subótima com equipas de CP especializadas, limitação temporal e horário laboral preenchido e incompreensão do papel do MF na prestação de CP. 5,61

Existem outras questões que devem ser alvo de reflexão na gestão e reconhecimento do delirium nos CSP, no- 
meadamente a heterogeneidade da acessibilidade aos CSP em Portugal e o isolamento e iliteracia da população idosa, que muitas vezes vive sozinha, o que pode levar ao reconhecimento tardio do quadro clínico pelo MF, quando este já se encontra numa fase avançada. $O$ acesso presencial aos CSP também está limitado, o que pode reforçar a necessidade de articular cuidados com equipas de CP hospitalares. Não obstante, não existem equipas de CP comunitárias em todo o território nacional que permitam uma eficiente cobertura, de forma a colmatar estas questões e articular eficazmente cuidados.

Existem, assim, muitas barreias para o reconhecimento desta entidade, apesar de a sua abordagem precoce ser manifestamente importante. É pelo impacto que o delirium tem nos serviços de saúde, profissionais, cuidadores e, sobretudo, no doente, que se devem reforçar as estratégias de prevenção, identificação atempada e intervenções para a sua gestão precoce, no contexto de CP na comunidade. Deve ser estimulada a promoção institucional, o desenvolvimento de protocolos e iniciativas de educação para a saúde, adaptadas ao nível de cuidados exigidos e à localização do doente na comunidade.

\section{RESPONSABILIDADES ÉTICAS}

CONFLITOS DE INTERESSE: Os autores declaram não possuir conflitos de interesse.

SUPORTE FINANCEIRO: O presente trabalho não foi suportado por nenhum subsídio ou bolsa.

PROVENIÊNCIA E REVISÃO POR PARES: Não comissionado; revisão externa por pares.

\section{ETHICAL DISCLOSURES}

CONFLICTS OF INTEREST: The authors have no conflicts of interest to declare.

FINANCIAL SUPPORT: This work has not received any contribution grant or scholarship.

PROVENANCE AND PEER REVIEW: Not commissioned; externally peer reviewed.

\section{REFERÊNCIAS}

1. Sepúlveda C, Marlin A, Yoshida T, Ullrich A. Palliative Care: The World Health Organization's global perspective. J Pain Symptom Manage. 2002;24:91-6.

2. Diário da República n. 172/2012, Série I de 2012-09-05, páginas 5119-5124.

3. Comissão Nacional de Cuidados Paliativos. Plano Estratégico para o Desenvolvimento dos Cuidados Paliativos: Biénio 2019-2020. [Online] https://www.sns.gov.pt/wp-content/uploads/2019/04/PEDCP-2019-2020-versao-final-10.02.2019.pdf.
4. Galriça Neto I. Palliative care development is well under way in Portugal. Eur J Palliat Care. 2010;17:278-81.

5. Rotar Pavlič D, Aarendonk D, Wens J, Rodrigues Simões JA, Lynch M, Murray S. Palliative care in primary care: European Forum for Primary Care position paper. Prim Health Care Res Dev. 2019;20:e133.

6. Desmedt M, Michel H. Palliative home care: improving co-operation between the specialist team and the family doctor. Support Care Cancer. 2002;10:343-8.

7. Barbosa A, Tavares F, Neto I. Manual de Cuidados Paliativos. $3^{2}$ ed. Lisboa: Faculdade de Medicina de Lisboa; 2016.

8. Agar M, Bush SH. Delirium at the End of Life. Med Clin North Am. 2020;104:491-501.

9. Kalish VB, Gillham JE, Unwin BK. Delirium in older persons: evaluation and management. Am Fam Physician. 2014;90:1508.

10. Wada T, Wada M, Wada M, Onishi H. Characteristics, interventions, and outcomes of misdiagnosed delirium in cancer patients. Palliat Support Care. 2010;8:125-31.

11. Agar MR, Quinn SJ, Crawford GB, Ritchie CS, Phillips JL, Collier A, et al. Predictors of Mortality for Delirium in Palliative Care. J Palliat Med. 2016;19:1205-9.

12. Breitbart W, Alici Y. Evidence-based treatment of delirium in patients with cancer. J Clin Oncol. 2012;30:1206-14.

13. LeGrand SB. Delirium in palliative medicine: a review. J Pain Symptom Manage. 2012;44:583-94.

14. Bond SM. Delirium at home: strategies for home health clinicians. Home Healthc Nurse. 2009;27:24-34; quiz 5-6.

15. Morita T, Akechi T, Ikenaga M, Inoue S, Kohara H, Matsubara T, et al. Terminal delirium: recommendations from bereaved families' experiences. J Pain Symptom Manage. 2007;34:57989.

16. Leslie DL, Inouye SK. The importance of delirium: economic and societal costs. J Am Geriatr Soc. 2011;59:S241-3.

17. MacLullich AMJ, Shenkin SD. Delirium research, education and practice. Age Ageing. 2019;48:619-23.

18. Bush SH, Kanji S, Pereira JL, Davis DHJ, Currow DC, Meagher $\mathrm{D}$, et al. Treating an established episode of delirium in palliative care: expert opinion and review of the current evidence base with recommendations for future development. J Pain Symptom Manage. 2014;48:231-48.

19. Hoek PD, Schers HJ, Hendriks JCM, Vissers KCP, Hasselaar JGJ. Palliative care consultations in primary care: a cross-sectional survey among Dutch general practitioners. BMJ Support Palliat Care. 2019;9:e30.

20. Gomes B, Higginson IJ, Calanzani N, Cohen J, Deliens L, Daveson BA, et al. Preferences for place of death if faced with advanced cancer: a population survey in England, Flanders, Germany, Italy, the Netherlands, Portugal and Spain. Ann Oncol. 2012;23:2006-15.

21. Prayce R, Quaresma F, Neto IG. Delirium: The 7th Vital Sign? Acta Med Port. 2018;31:51-8.

22. Danielsen BV, Sand AM, Rosland JH, Førland O. Experiences and challenges of home care nurses and general practitioners in home-based palliative care - a qualitative study. BMC Palliat Care. 2018;17:95.

23. American Psychiatric Association: Diagnostic and Statistical Manual of Mental Disorders. 5th ed. Arlington: American Psychiatric Association; 2013.

24. Gaertner J, Eychmueller S, Leyhe T, Bueche D, Savaskan E, Schlogl M. Benzodiazepines and/or neuroleptics for the treat- 
ment of delirium in palliative care?-a critical appraisal of recent randomized controlled trials. Ann Palliat Med. 2019;8:504-15.

25. National Clinical Guideline Center. National Institute for Health and Clinical Excellence: Guidance. Delirium: Diagnosis, Prevention and Management. London: Royal College of Physicians; 2010.

26. Caraceni A, Simonetti F. Palliating delirium in patients with cancer. Lancet Oncol. 2009;10:164-72.

27. Keeley PW. Delirium at the end of life. BMJ Clin Evid. 2009;2009

28. Mercadante S, Masedu F, Balzani I, De Giovanni D, Montanari L, Pittureri C, et al. Prevalence of delirium in advanced cancer patients in home care and hospice and outcomes after 1 week of palliative care. Support Care Cancer. 2018;26:913-9.

29. Watt CL, Momoli F, Ansari MT, Sikora L, Bush SH, Hosie A, et al. The incidence and prevalence of delirium across palliative care settings: A systematic review. Palliat Med. 2019;33:865-77.

30. Hey J, Hosker C, Ward J, Kite S, Speechley H. Delirium in palliative care: Detection, documentation and management in three settings. Palliat Support Care. 2015;13:1541-5.

31. Inouye SK, Westendorp RG, Saczynski JS. Delirium in elderly people. Lancet. 2014;383:911-22.

32. Bruera E, Miller L, McCallion J, Macmillan K, Krefting L, Hanson J. Cognitive failure in patients with terminal cancer: a prospective study. J Pain Symptom Manage. 1992;7:192-5.

33. Lawlor PG, Bush SH. Delirium diagnosis, screening and management. Curr Opin Support Palliat Care. 2014;8:286-95.

34. Magny E, Le Petitcorps H, Pociumban M, Bouksani-Kacher Z, Pautas É, Belmin J, et al. Predisposing and precipitating factors for delirium in community-dwelling older adults admitted to hospital with this condition: A prospective case series. PLoS One. 2018;13:e0193034.

35. Pérez-Ros P, Martínez-Arnau FM, Baixauli-Alacreu S, Caballero-Pérez M, García-Gollarte JF, Tarazona-Santabalbina F. Delirium Predisposing and Triggering Factors in Nursing Home Residents: A Cohort Trial-Nested Case-Control Study. J Alzheimers Dis. 2019;70:1113-22.

36. Lawlor PG, Bush SH. Delirium in patients with cancer: assessment, impact, mechanisms and management. Nat Rev Clin Oncol. 2015;12:77-92.

37. Inouye SK. Delirium in older persons. N Engl J Med. 2006;354:1157-65.

38. Grassi L, Caraceni A, Mitchell AJ, Nanni MG, Berardi MA, Caruso R, et al. Management of delirium in palliative care: a review. Curr Psychiatry Rep. 2015;17:550.

39. Hui D. Delirium in the palliative care setting: "Sorting" out the confusion. Palliat Med. 2019;33:863-4.

40. National Institute for Health and Care Excellence: Clinical Guidelines. Delirium: prevention, diagnosis and management. London: National Institute for Health and Care Excellence; 2019.

41. Spiller JA, Keen JC. Hypoactive delirium: assessing the extent of the problem for inpatient specialist palliative care. Palliat Med. 2006;20:17-23.

42. Mercadante S, Masedu F, Maltoni M, De Giovanni D, Montanari L, Pittureri C, et al. Symptom expression in advanced cancer patients admitted to hospice or home care with and without delirium. Intern Emerg Med. 2019;14:515-20.

43. Bush SH, Tierney S, Lawlor PG. Clinical Assessment and Management of Delirium in the Palliative Care Setting. Drugs. 2017;77:1623-43.
44. Burry L, Hutton B, Williamson DR, Mehta S, Adhikari NK, Cheng W, et al. Pharmacological interventions for the treatment of delirium in critically ill adults. Cochrane Database Syst Rev. 2019;9:Cd011749.

45. Teale EA, Munyombwe T, Schuurmans M, Siddiqi N, Young J. A prospective observational study to investigate utility of the Delirium Observational Screening Scale (DOSS) to detect delirium in care home residents. Age Ageing. 2018;47:56-61.

46. Clary PL, Lawson P. Pharmacologic pearls for end-of-life care. Am Fam Physician. 2009;79:1059-65.

47. Wong CL, Holroyd-Leduc J, Simel DL, Straus SE. Does this patient have delirium?: value of bedside instruments. JAMA. 2010;304:779-86.

48. Mitchell AJ, Shukla D, Ajumal HA, Stubbs B, Tahir TA. The Mini-Mental State Examination as a diagnostic and screening test for delirium: systematic review and meta-analysis. Gen Hosp Psychiatry. 2014;36:627-33.

49. Ryan K, Leonard M, Guerin S, Donnelly S, Conroy M, Meagher D. Validation of the confusion assessment method in the palliative care setting. Palliat Med. 2009;23:40-5.

50. Dosa D, Intrator O, McNicoll L, Cang Y, Teno J. Preliminary derivation of a Nursing Home Confusion Assessment Method based on data from the Minimum Data Set. J Am Geriatr Soc. 2007;55:1099-105.

51. Brajtman S, Wright D, Hogan DB, Allard P, Bruto V, Burne D, et al. Developing guidelines on the assessment and treatment of delirium in older adults at the end of life. Can Geriatr J. 2011;14:40-50.

52. Barbosa A, Tavares F, Neto I. Manual de Cuidados Paliativos. $3^{a}$ ed. Lisboa: Faculdade de Medicina de Lisboa; 2016.

53. Direção Geral de Saúde. Orientação nº21/2011: Prevenção de comportamentos dos doentes que põem em causa a sua segurança ou da sua envolvente. Lisboa: DGS; 2011.

54. Okuyama T, Yoshiuchi K, Ogawa A, Iwase S, Yokomichi N, Sakashita A, et al. Current Pharmacotherapy Does Not Improve Severity of Hypoactive Delirium in Patients with Advanced Cancer: Pharmacological Audit Study of Safety and Efficacy in Real World (Phase-R). Oncologist. 2019;24:e574-e82.

55. Agar MR, Lawlor PG, Quinn S, Draper B, Caplan GA, Rowett D, et al. Efficacy of Oral Risperidone, Haloperidol, or Placebo for Symptoms of Delirium Among Patients in Palliative Care: A Randomized Clinical Trial. JAMA Intern Med. 2017;177:3442.

56. Devlin JW, Roberts RJ, Fong JJ, Skrobik Y, Riker RR, Hill NS, et al. Efficacy and safety of quetiapine in critically ill patients with delirium: a prospective, multicenter, randomized, double-blind, placebo-controlled pilot study. Crit Care Med. 2010;38:419-27.

57. Direção Geral de Saúde. Norma n 009/2018 de 06/04/2018: Fármacos e Materiais de Consumo Clínico na Prestação de Cuidados Paliativos Domiciliários. Lisboa: DGS; 2018.

58. Hui D, Frisbee-Hume S, Wilson A, Dibaj SS, Nguyen T, De La Cruz M, et al. Effect of Lorazepam With Haloperidol vs Haloperidol Alone on Agitated Delirium in Patients With Advanced Cancer Receiving Palliative Care: A Randomized Clinical Trial. JAMA. 2017;318:1047-56.

59. van Eijk MM, Roes KC, Honing ML, Kuiper MA, Karakus A, van der Jagt $M$, et al. Effect of rivastigmine as an adjunct to usual care with haloperidol on duration of delirium and mortality in critically ill patients: a multicentre, double-blind, placebo-controlled randomised trial. Lancet. 2010;376:1829-37.

60. Senior H, Grant M, Rhee JJ, Aubin M, McVey P, Johnson C, et al. General practice physicians' and nurses' self-reported 
multidisciplinary end-of-life care: a systematic review. BMJ Support Palliat Care. 2019; bmjspcare-2019-001852. doi: 10.1136/bmjspcare-2019-001852.

61. Malik S, Goldman R, Kevork N, Wentlandt K, Husain A, Merrow N, et al. Engagement of Primary Care Physicians in Home Palliative Care. J Palliat Care. 2017;32:3-10. 